\title{
Role of pulmonary function in the detection of allograft dysfunction after heart-lung transplantation
}

\author{
Alain Van Muylem, Christian Mélot, Martine Antoine, Christiane Knoop, \\ Marc Estenne
}

\begin{abstract}
Background - Lung function is altered by infection and rejection in patients who undergo heart-lung transplantation. The sensitivity, specificity, and positive/negative predictive values (PPV and NPV) of lung function for the detection of allograft dysfunction in these patients were measured. Methods - Thirty three patients who underwent heart-lung transplantation were followed for a mean period of $\mathbf{1 6 . 3}$ months. On 123 occasions functional measurements were obtained at the time a transbronchial biopsy specimen and/or bronchoalveolar lavage fluid was taken, which were used as gold standards. Optimal sensitivity (the value for which sensitivity equals specificity) was computed for each functional test from receiveroperator characteristic (ROC) curves.
\end{abstract}

Results - Acute rejection was present on 31 occasions and infection on 36 occasions; 56 samples were normal. Infection and rejection were accompanied by airflow obstruction, a rise in the slopes of the alveolar plateaus for nitrogen, hexafluoride sulphur and helium $\left(\mathbf{S N}_{2}, \mathbf{S s F}_{6}\right.$, and $\left.\mathrm{SHe}_{\mathrm{H}}\right)$, and a decrease in the difference between $\mathbf{S S F}_{6}$ and SHe $(\Delta S)$, total lung capacity (TLC), and lung transfer factor (TLCO). Optimal sensitivities for SHe, mid forced expiratory flow $\left(\mathbf{F E F}_{25-75}\right)$, TLC, and forced expiratory volume in one second $\left(\mathrm{FEV}_{1}\right)$ were $68 \%$, $67 \%, 66 \%$, and $60 \%$, respectively; they were not different for infection and rejection and did not change over the study period. For infection and rejection together, PPV ranged from $72 \%$ to $88 \%$ and NPV from $27 \%$ to $52 \%$ according to the functional test and the postoperative period considered. Conclusions - Indices of ventilation distribution, $\mathrm{FEF}_{25-75}$, and TLC have the best optimal sensitivity for the diagnosis of infection and rejection after heart-lung transplantation. The high PPV of pulmonary function in detecting allograft dysfunction observed in this study suggests that a diagnostic procedure should be performed whenever one or more functional tests deteriorate; conversely, the low NPV indicates that a stable pulmonary function does not rule out allograft dysfunction.

(Thorax 1997;52:643-647)

Keywords: lung transplantation, pulmonary function, infection, rejection.
Lung transplantation has become an accepted therapeutic option for patients with various end-stage vascular and parenchymal lung diseases. ${ }^{1}$ Outcome after transplantation has improved with advances in operative techniques but optimal management of infection and rejection remains a problem, partly because these complications may be undetectable by clinical evaluation. $^{23}$ Bronchoscopy with bronchoalveolar lavage and transbronchial lung biopsy has been widely utilised to diagnose allograft dysfunction $^{4-6}$ but this procedure is invasive and therefore cannot be used as a screening test.

Lung function in recipients of heart-lung and double-lung transplants is altered by infection and rejection which produce transient decreases in forced expiratory volume in one second $\left(\mathrm{FEV}_{1}\right)$ and forced vital capacity (FVC) ${ }^{4-10}$ On this basis, it has been suggested that monitoring lung function may aid early detection of allograft dysfunction and may help to decide when a bronchoscopy is required. Values of sensitivity and specificity of lung function for the diagnosis of infection and rejection after heart-lung transplantation have been provided in a previous work ${ }^{9}$ but the only variable studied was $\mathrm{FEV}_{1}$. In the present study we have prospectively investigated the sensitivity, specificity, and positive/negative predictive value of a variety of functional variables in 33 heart-lung transplant patients followed over a 43 month period. In addition to standard pulmonary function, we have measured indices of ventilation distribution which provide information about mixing in the periphery of the lung ${ }^{11}$ and hence might be more sensitive than standard pulmonary function to allograft dysfunction. ${ }^{12}$

\section{Methods}

Thirty three non-smoking heart-lung transplant patients $(20$ men) aged nine to 62 years were studied between January 1992 and July 1995. Ten patients had received transplants between two months and three years before the start of the study; the others were given transplants during the course of the study and were enrolled consecutively. Transplantation was performed for primary pulmonary hypertension in 13 patients, cystic fibrosis in 11 patients, Eisenmenger's syndrome in five patients, bronchiectasis in two, and emphysema and coal miner pneumoconiosis in the remaining two patients. Patients who were 
considered to have chronic rejection were not included in the study. Bronchiolitis obliterans syndrome (BOS) was defined histologically by the presence of obliterative bronchiolitis or functionally by an irreversible decline of more than $20 \%$ in $\mathrm{FEV}_{1}$ compared with the best postoperative value. If the diagnosis of BOS was made after enrollment into the study, only data pertinent to the period preceding this diagnosis were included in the data analysis. All patients gave verbal informed consent to the procedures as approved by the Human Studies Committee of our institution.

Diagnostic bronchoscopic examination was performed whenever patients developed symptoms such as fever, purulent sputum production or increased dyspnoea, physical signs, radiological abnormalities, or more than a $10 \%$ decline in home spirometric values, confirmed by formal spirometry. If there was a strong clinical suspicion of infection with purulent secretions at endobronchial examination, bronchoalveolar lavage (BAL) without transbronchial biopsy (TBB) was initially performed; if Gram stains and cultures were negative or if patients did not respond to antibiotic treatment, a second bronchoscopy with BAL and TBB was carried out. Surveillance bronchoscopy was defined as a procedure performed according to a predefined protocol in recipients who were free of new clinical symptoms and had stable lung function and chest radiography. Surveillance bronchoscopy was performed at one, three, and six months after surgery and annually thereafter. Bronchoscopy was also performed 3-4 weeks after the diagnosis of acute rejection to assess the response to treatment. Surveillance and follow up bronchoscopic examinations always included both BAL and TBB.

Fibreoptic bronchoscopy was performed with local anaesthesia and BAL fluid and TBB specimens were taken and processed following standard procedures. At least six biopsy specimens were taken and serial sections were graded for acute rejection by a single pathologist using the guidelines established by the International Society for Heart and Lung Transplantation. ${ }^{13}$ The diagnosis of acute cellular rejection was considered only when the TBB specimens were graded $\mathrm{A}_{2}$ or $\mathrm{A}_{3}$; lymphocytic bronchitis was defined as the presence of peribronchial lymphocytic infiltrates. Cytomegalovirus (CMV) infection was defined as isolation of CMV from cultures of BAL fluid and CMV pneumonia as the presence of characteristic CMV associated histological changes on TBB specimens or the isolation of CMV in the BAL fluid in the presence of an infiltrate which resolved with antiviral therapy. The diagnosis of bacterial pneumonia was made from positive cultures of BAL fluid in the presence of a new radiological infiltrate that responded favourably to antibiotics. Bacterial bronchitis was diagnosed from positive cultures of BAL fluid with increased sputum production in the absence of any new radiological abnormality.

Measurements of static lung volumes and airway resistance were obtained within the 2-3 days before the bronchoscopic examination with the patient seated in a constant volume body plethysmograph. Measurements of FVC, $\mathrm{FEV}_{1}$, maximal expiratory flow rates, and carbon monoxide transfer factor (TLCO) were made using a Sensormedics 2400 unit (Sensormedics, Anaheim, California, USA). For the single breath washout test subjects were connected to a double bag-in-box system through a non-rebreathing valve with a $20 \mathrm{ml}$ instrumental dead space. They inhaled a gas mixture containing $5 \%$ helium $(\mathrm{He}), 5 \%$ hexafluoride sulphur $\left(\mathrm{SF}_{6}\right)$, and $90 \% \mathrm{O}_{2}$ from functional residual capacity (FRC) to one litre above $\mathrm{FRC}^{14}$ and then expired at a constant flow of $0.4 \mathrm{l} / \mathrm{s}$. Flow was measured with a Lilly-type pneumotachograph connected to a differential pressure transducer, and volume was obtained by integration of the flow signal. Because the pneumotachograph was located in the wall of the bag-in-box system, flow was independent of gas composition. Gases were sampled at the mouth and concentrations of $\mathrm{He}, \mathrm{SF}_{6}$, and $\mathrm{N}_{2}$ were analysed using a quadrupole mass spectrometer (Centronic 200, MGA) which incorporated an automatic control of the sensitivity maintaining the sum of inspired and expired concentrations $\left(\mathrm{He}, \mathrm{SF}_{6}\right.$, $\mathrm{O}_{2}, \mathrm{~N}_{2}, \mathrm{CO}_{2}$ ) at $100 \%$. By excluding water vapour from the sum, we expressed gas concentration as a percentage of total dry gas. The slope of the alveolar plateau for each gas $\left(\mathrm{SN}_{2}\right.$, $\mathrm{SsF}_{6}, \mathrm{SHe}$ ) was obtained from a linear regression analysis performed at $35-80 \%$ of the expired volume. The downgoing $\mathrm{He}$ and $\mathrm{SF}_{6}$ slopes were treated as positive, as were upgoing $\mathrm{N}_{2}$ slopes. The difference between the $\mathrm{SF}_{6}$ and $\mathrm{He}$ slopes $(\Delta S)$ was also calculated. All signals were sampled at $50 \mathrm{~Hz}$ and stored in an Olivetti PC for subsequent analysis.

\section{DATA ANALYSIS}

The analysis included all episodes of infection and rejection that met the following criteria: (1) a diagnosis based on TBB and/or BAL fluid specimens; and (2) functional tests, including indices of ventilation distribution, measured within the 2-3 day period before the bronchoscopic examination. Because we used BAL fluid/TBB specimens as the gold standard, episodes of rejection that responded functionally to pulsed steroid or cytolytic therapy but for which TBB specimens were either not available, non-diagnostic, or only showed lesions graded $A_{1}$ were not included in the data analysis.

For each functional test the receiver-operator characteristic (ROC) curve $\mathrm{e}^{15}$ was computed by plotting sensitivity (true positive rate) on the $y$ axis against 1 - specificity (false positive rate) on the $x$ axis for different cutoff values. These values were defined in three different ways as percentages of predicted values, percentages of best postoperative values, and percentages of the last values obtained before the acute event. The optimal sensitivity was given by the intersection between the ROC curve and the second bisectrix - that is, the value for which sensitivity equals specificity. Optimal sensitivity was computed for each functional variable 
Table 1 Results of examination of bronchoalveolar lavage fluid and transbronchial biopsy specimens

\begin{tabular}{|c|c|c|c|c|c|c|c|}
\hline & \multirow{2}{*}{$\begin{array}{l}\text { Acute cellular } \\
\text { rejection }\end{array}$} & \multirow{2}{*}{$\begin{array}{l}\text { Lymphocytic } \\
\text { bronchitis }\end{array}$} & \multicolumn{2}{|c|}{ Pneumonia } & \multirow{2}{*}{$\begin{array}{l}\text { Bacterial } \\
\text { bronchitis }\end{array}$} & \multirow[t]{2}{*}{ Normal } & \multirow[t]{2}{*}{ Total } \\
\hline & & & Bacterial & Viral & & & \\
\hline Routine & 3 & 4 & - & - & - & 34 & 41 \\
\hline Clinically indicated & 8 & 6 & $17^{*}$ & $2^{* *}$ & 17 & 6 & 56 \\
\hline Follow up & 7 & 3 & - & - & - & 16 & 26 \\
\hline Total & 18 & 13 & 17 & 2 & 17 & 56 & 123 \\
\hline
\end{tabular}

* One pneumonia was bacterial and fungal.

** One pneumonia was bacterial and viral.

Table 2 Mean (SD) changes in pulmonary function of 33 heart-lung transplant patients during 67 episodes of infection and rejection

\begin{tabular}{lcllc}
\hline & $\begin{array}{l}\text { Best postoperative } \\
\text { value }(n=33)\end{array}$ & $\begin{array}{l}\text { All acute events } \\
(n=67)\end{array}$ & $\begin{array}{l}\text { Rejection } \\
(n=31)\end{array}$ & $\begin{array}{l}\text { Infection } \\
(n=36)\end{array}$ \\
\hline FEV $_{1}(\%$ pred $)$ & $100.2(17.2)$ & $83.7(20.3)^{* * *}$ & $82.7(18.5)^{* * * *}$ & $84.5(21.9)^{* * *}$ \\
VC (\% pred) & $99.2(18.9)$ & $86.5(20.4)^{* *}$ & $82.5(20.4)^{* *}$ & $90.0(19.3)^{*}$ \\
FEF $_{25-75}(\%$ pred $)$ & $106.9(26.2)$ & $73.7(25.1)^{* * *}$ & $78.5(23.9)^{* * *}$ & $69.6(25.7)^{* * *}$ \\
TLCO (\% pred) & $70.5(16.6)$ & $50.7(15.3)^{* * *}$ & $49.0(13.9)^{* * *}$ & $52.1(16.4)^{* * *}$ \\
TLC (\% pred) & $109.2(13.9)$ & $97.4(16.4)^{* * *}$ & $91.4(14.5)^{* * *}$ & $102.8(16.3)^{*}$ \\
sRaw (cm H $\left.\mathrm{H}_{2} \mathrm{O}\right)$ & $1.59(1.44)$ & $4.3(2.4)^{* * *}$ & $3.6(2.1)^{* * *}$ & $4.9(2.5)^{* * *}$ \\
$\mathrm{SHE}(\% / 1)$ & $2.66(1.38)$ & $5.18(4.69)^{* * *}$ & $5.16(4.69)^{* * *}$ & $5.20(3.22)^{* * *}$ \\
$\Delta \mathrm{S}(\% / 1)$ & $1.29(0.94)$ & $-0.03(1.42)^{* * *}$ & $-0.16(1.69)^{* * *}$ & $0.07(1.42)^{* * *}$ \\
\hline
\end{tabular}

$\mathrm{FEV}_{1}=$ forced expiratory volume in one second; $\mathrm{VC}=$ vital capacity; $\mathrm{FEF}_{25-75}=$ mid-expiratory flow rate between $25 \%$ and $75 \% \mathrm{VC}$; TLCO = carbon monoxide transfer factor; TLC = total lung capacity; sRaw = specific airway resistance; SHE = slope of the alveolar plateau for helium; $\Delta \mathrm{S}=$ difference in slopes of the $\mathrm{SF}_{6}$ and He alveolar plateaus.

$* \mathrm{p}<0.05 ; * * \mathrm{p}<0.01 ; * * * \mathrm{p}<0.001$ compared with best postoperative value.

using the three different baselines. Positive predictive values (PPV) were calculated as the ratio of true positive/all positive tests and negative predictive values (NPV) as the ratio of true negative/all negative tests. However, because predictive values are inherently influenced by prevalence, we calculated the prevalence of infection and rejection in our population between postoperative months 1-6, 7-12, and 13-24 and then computed PPV and NPV using the Bayes' theorem. Prevalence was defined as the percentage of patients presenting with one or more acute episodes of infection or rejection during the postoperative period considered.

Predicted values for static and dynamic lung volumes and TLCo were derived from the ECCS Working Party regression equations. ${ }^{16}$ Statistical analysis was made using paired Wilcoxon tests and the level of statistical significance was taken as $\mathrm{p}<0.05$. All data are reported as mean (SD).

\section{Results}

The 33 patients were followed for a mean period of 16.3 months (range 0.5-36) during which a total of 184 bronchoscopic examinations were performed. On 126 occasions a complete functional assessment could be obtained within the $2-3$ days preceding the bronchoscopy but this proved to be impossible on 58 occasions, either because the patient's clinical condition did not allow testing or for logistic reasons. Of the 126 bronchoscopic examinations, three were excluded from analysis because the TBB specimen revealed only grade $A_{1}$ rejection (see Methods). A total of 123 events were thus included, for which both BAL fluid and TBB specimens were available on 89 occasions and BAL fluid alone on 34 occasions.

Of the routine surveillance biopsy specimens taken, 34 of 41 were normal (table 1) and seven revealed unsuspected rejection. Of the 56

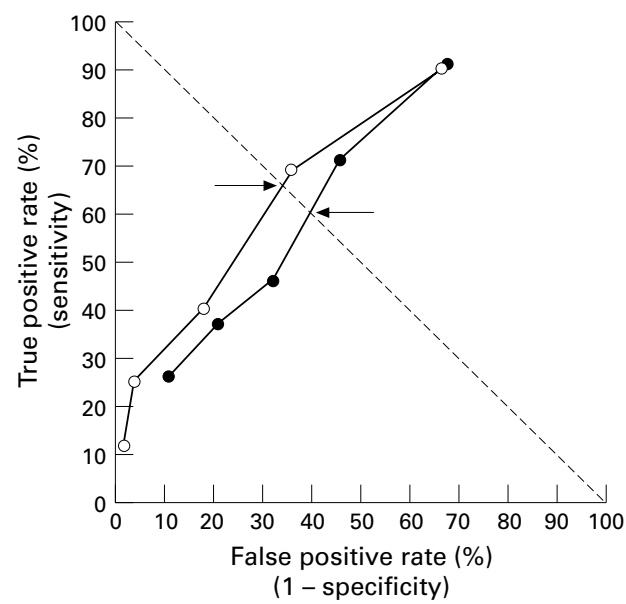

Figure 1 Receiver-operator characteristic (ROC) curve for FEV $V_{1}(\mathbf{O})$ and TLC (O) in 33 heart-lung transplant patients who were studied on 123 occasions. The circles represent sensitivity and 1 - specificity for cutoff values corresponding to $95 \%, 90 \%, 85 \%, 80 \%$, and $75 \%$ of the best postoperative value. The dotted line gives values of optimal sensitivity (shown by the arrows) - that is, values for which sensitivity equals specificity.

clinically indicated BAL fluid/TBB specimens examined only six were normal, while the rest showed rejection $(\mathrm{n}=14)$ or infection $(\mathrm{n}=$ 36). On 17 occasions the infection was a bacterial bronchitis and on 19 occasions a pneumonia, either bacterial $(n=16)$, viral $(n=1)$, bacterial and fungal $(n=1)$, or bacterial and viral $(n=1)$. Of the $26 \mathrm{BAL}$ fluid/TBB specimens taken during follow up, 16 were normal and 10 showed ongoing rejection.

Table 2 shows the mean changes in pulmonary function at the time of an abnormal BAL fluid/TBB specimen compared with the best postoperative value. On average, the best postoperative values were within normal limits for all tests except for a slight reduction in TLCo. Infection and rejection produced significant changes in all functional variables: $\mathrm{FEV}_{1}, \mathrm{VC}, \mathrm{FEF}_{25-75}$, TLCO, TLC, $\mathrm{FEV}_{1} / \mathrm{VC}$ (not shown in the table) decreased whereas specific airways resistance (sRaw) and $\mathrm{SHe}, \mathrm{SN}_{2}$, and $\mathrm{SsF}_{6}$ (not shown in the table) increased. Of interest, $\Delta S$ decreased and became slightly negative due to a greater rise in $\mathrm{SHe}$ than in $\mathrm{SsF}_{6}$. The changes were not significantly different for the two types of events.

Figure 1 shows typical ROC curves for TLC and $\mathrm{FEV}_{1}$. Each point on the figure represents sensitivity and 1 - specificity for a cutoff value corresponding to $95 \%, 90 \%, 85 \%, 80 \%$, and $75 \%$ of the best postoperative value. The intersection of the ROC curves with the second bisectrix gives the optimal sensitivity which amounted to $66 \%$ for TLC and $60 \%$ for $\mathrm{FEV}_{1}$.

Table 3 shows the values of optimal sensitivity for all functional tests in detecting any acute event, and rejection and infection separately. The best values were found for indices of ventilation distribution followed by $\mathrm{FEF}_{25-75}$ TLC, and $\mathrm{FEV}_{1}$. Optimal sensitivities were not different for infection and rejection and they did not change according to the time elapsed since surgery. Similarly, expressing data as a 
Table 3 Optimal sensitivity of pulmonary function tests measured on 123 occasions in 33 heart-lung transplant patients

\begin{tabular}{llll}
\hline & $\begin{array}{l}\text { All acute events } \\
(n=67)\end{array}$ & $\begin{array}{l}\text { Rejection } \\
(n=31)\end{array}$ & $\begin{array}{l}\text { Infection } \\
(n=36)\end{array}$ \\
\hline SHe & 68 & 64 & 70 \\
$\Delta \mathrm{S}$ & 67 & 66 & 68 \\
$\mathrm{FEF}_{25-75}$ & 67 & 62 & 73 \\
$\mathrm{TLC}$ & 66 & 69 & 65 \\
$\mathrm{FEV}_{1}$ & 60 & 61 & 62 \\
sRaw $_{\text {VC }}$ & 60 & 61 & 58 \\
TLCo & 57 & 60 & 54 \\
\hline
\end{tabular}

For definition of abbreviations see footnote to table 2 . Tests are ranked by order of decreasing sensitivity, expressed in $\%$

Table 4 Prevalence of infection and rejection and positive and negative predictive values for all functional tests according to postoperative period (in months) and diagnosis

\begin{tabular}{llll}
\hline & $1-6$ months & $7-12$ months & 13-24 months \\
\hline Prevalence & & & \\
All events & 78 & 67 & 67 \\
Rejection & 61 & 25 & 13 \\
Infection & 30 & 58 & 60 \\
$\quad$ & & \\
Positive predictive value & & \\
All events & $82-88$ & $72-81$ & $72-81$ \\
Rejection & $65-78$ & $29-42$ & $16-25$ \\
Infection & $33-54$ & $61-76$ & $63-81$ \\
Negative predictive value & & \\
All events & $27-37$ & $40-52$ & $40-52$ \\
Rejection & $43-59$ & $78-86$ & $89-94$ \\
Infection & $73-86$ & $46-63$ & $43-64$ \\
\hline
\end{tabular}

percentage of the best postoperative value or as a percentage of the last value measured before the acute event did not significantly alter values of optimal sensitivity. In contrast, these values were significantly lower $(\mathrm{p}<0.05)$ when percentages of predicted values were used.

Table 4 gives the prevalence of acute events, infection, and rejection calculated for the three
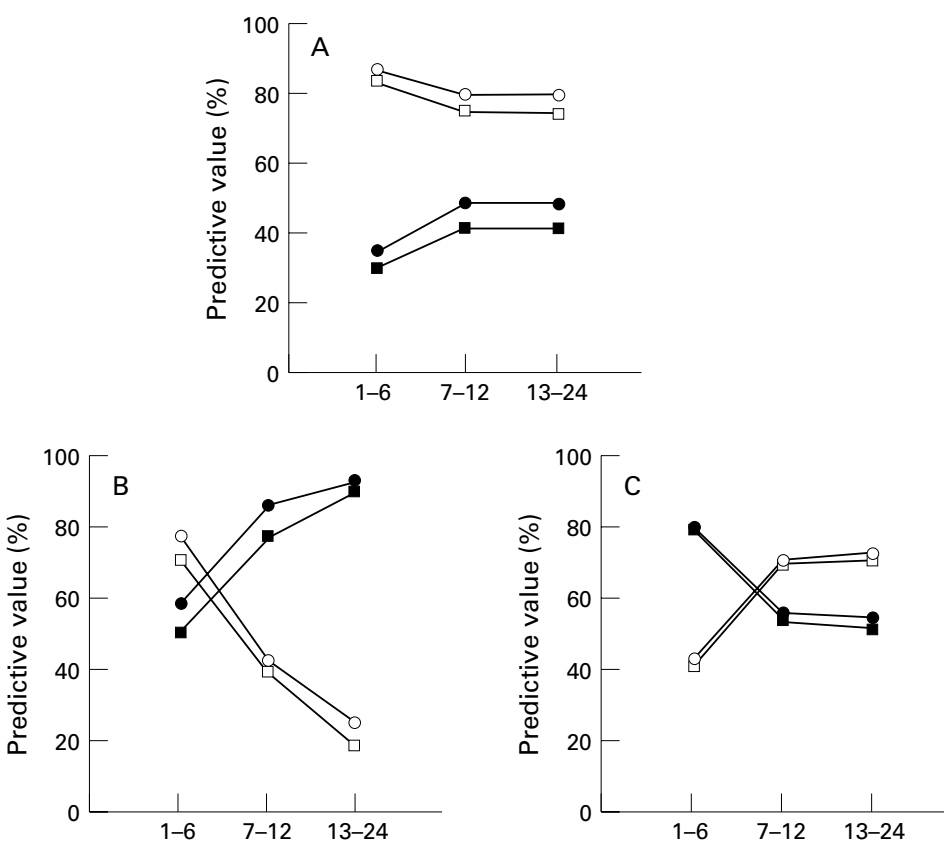

Figure 2 Positive and negative predictive values of $F E V_{1}(\square, \square)$ and TLC (O, for the diagnosis of $(A)$ an acute episode of allograft dysfunction, (B) rejection, and $(C)$ infection 1-6, 7-12, and 13-24 months after surgery. postoperative periods considered and ranges of positive and negative predictive values computed for all functional tests using the optimal sensitivities shown in table 3; fig 2 displays PPV and NPV for $\mathrm{FEV}_{1}$ and TLC. When all acute events were analysed together the PPV was high and NPV low, whatever the variable and postoperative period considered. However, for the diagnosis of rejection NPV increased and PPV decreased substantially after the sixth postoperative month. An inverse trend was noted for the diagnosis of infection. Because optimal sensitivities did not change according to the time elapsed since surgery, changes in PPV and NPV with time were due to changes in prevalence.

\section{Discussion}

This study confirms that acute infection and rejection of the lung allograft alter pulmonary function. The 67 episodes studied here were accompanied by airflow obstruction characterised by a fall in $\mathrm{FEV}_{1}, \mathrm{FEV}_{1} / \mathrm{VC}$, and $\mathrm{FEF}_{25-75}$ and a rise in sRaw. There was also a significant reduction in TLC and TLCO. This pattern of changes is very similar to that reported in earlier studies by our group ${ }^{12}$ and others. ${ }^{4-10}$ The present study also confirms our previous findings on the effect of allograft dysfunction on the distribution of ventilation. ${ }^{12}$ Infection and rejection produced consistent rises in the slope of the alveolar plateau for $\mathrm{N}_{2}$, $\mathrm{SF}_{6}$, and $\mathrm{He}$ and a fall in the slope difference between $\mathrm{SF}_{6}$ and $\mathrm{He}$. These changes suggest that both complications preferentially alter the distribution of ventilation in the periphery of the lung - that is, at the level of membranous and respiratory bronchioles. ${ }^{112}$

Only one earlier study by Otulana et $a l^{9}$ has assessed the sensitivity and specificity of lung function for the diagnosis of infection and rejection after heart-lung transplantation. These authors analysed 61 transbronchial biopsy specimens from 34 recipients. Using a 5\% fall in $\mathrm{FEV}_{1}$ as physiologically significant, they reported a sensitivity of $86 \%$ and $75 \%$ for the diagnosis of rejection before and after the third postoperative month, respectively; specificity was $84 \%$. For the diagnosis of infection $\mathrm{FEV}_{1}$ had a $75 \%$ sensitivity. In the present study a $5 \%$ fall in $\mathrm{FEV}_{1}$ had a $90 \%$ sensitivity in detecting infection or rejection, which is slightly greater than the value reported by Otulana et al. ${ }^{9} \mathrm{On}$ the other hand, we found a much lower value for specificity of only $35 \%$. This difference may be due to the fact that in the study by Otulana et al only 19 of the $61 \mathrm{BAL}$ fluid/TBB specimens taken were normal ${ }^{9}$ whereas in our study this ratio was 56/123 (table 1). Values of sensitivity and specificity are likely to be more accurate when the number of positive and negative events is balanced.

We elected to assess optimal sensitivity from ROC curves because this is the only method which allows comparison of the sensitivity and specificity of different functional tests. As shown in table 3, when all events were considered together the best optimal sensitivity was found for indices of ventilation distribution and 
$\mathrm{FEF}_{25-75}$. Of interest TLC, which is easier to measure than ventilation distribution, did almost as well as these variables in predicting an acute event, and proved to have the best optimal sensitivity in predicting rejection. Optimal sensitivity for $\mathrm{FEV}_{1}$ and TLCO was slightly lower, both for the diagnosis of rejection and infection. It should be stressed, however, that almost all infectious episodes included in this study were bacterial (table 1). The data presented in table 3 may not therefore apply to the detection of non-bacterial opportunistic infections for which functional tests such as TLCO might have a better sensitivity and specificity.

To assess the clinical usefulness of pulmonary function in the individual patient we computed positive and negative predictive values according to the time elapsed since surgery. When all events were considered together, PPV was high $(72-88 \%)$ throughout the study period with little difference between the variables tested - that is, a functional deterioration was generally predictive of allograft dysfunction. Accordingly, a diagnostic procedure should be performed when one or more functional variables deteriorate. In contrast, NPV was low $(27-52 \%)$ across the board which indicates that stable pulmonary function does not rule out allograft dysfunction.

In this study we used BAL fluid/TBB specimens as the gold standard. It has been suggested that TBB specimens may have a $15-28 \%$ false negative rate for rejection. ${ }^{35}$ However, we elected not to include lung rejection diagnosed on pulmonary function tests because these were the primary variables being studied; clinical criteria other than pulmonary function were not used either because lung rejection is very often asymptomatic, particularly after the early postoperative period. ${ }^{2}$ It is possible therefore that the actual rate of rejection was somewhat underestimated in this study, but we cannot determine how this might have affected our values of sensitivity and specificity.

To summarise, we have shown that indices of ventilation distribution, $\mathrm{FEF}_{25-75}$, and TLC have the best optimal sensitivity for the diagnosis of infection and rejection after heartlung transplantation. This suggests that, in addition to monitoring $\mathrm{FEV}_{1}$, the optimal functional follow up of lung transplant recipients may include sequential measurements of TLC,
$\mathrm{FEF}_{25-75}$, and, if possible, indices of ventilation distribution. The PPV for the detection of an acute event is high for all functional tests during the first two postoperative years, indicating that a diagnostic procedure is mandatory when one or more functional variables deteriorate. On the other hand, stable pulmonary function does not rule out allograft dysfunction.

The authors are indebted to Christine Piesen for preparation of the manuscript.

1 American Thoracic Society. Lung transplantation: report of the ATS workshop on lung transplantation. Am Rev Respir Dis 1993;147:772-6.

2 Guilinger RA, Paradis IL, Dauber JH, Yousem SA, Williams PA, Keenan RJ, et al. The importance of bronchoscopy with transbronchial biopsy and bronchoalveolar lavae in with transbronchial biopsy and bronchoalveolar lavage in the management of lung transplant

3 Pomerance A, Madden B, Burke MM, Yacoub MH. Transbronchial biopsy in heart and lung transplantation: clinicopathologic correlations. F Heart Lung Transplant 1995; opathologic

4 Starnes VA, Theodore J, Oyer PE, Billingham ME, Sibley RK, Berry G, et al. Evaluation of heart-lung transplan recipients with prospective, serial transbronchial biopsies and pulmonary function studies. F Thorac Cardiovasc Surg 1989;98:683-90.

5 Trulock EP, Ettinger NA, Brunt EM, Pasque MK, Kaiser LR, Cooper JD. The role of transbronchial lung biopsy in the treatment of lung transplant recipients. An analysis of 200 consecutive procedures. Chest 1992;102:1049-54.

6 Sibley RK, Berry GJ, Tazelaar HD, Kramer MR, Theodore J, Marshall SE, et al. The role of transbronchial biopsies in the management of lung transplant recipients. F Heart Lung Transplant 1993;12:308-24.

7 Penketh ARL, Higenbottam TW, Hutter J, Coutts C, Stewart $\mathrm{S}$, Wallwork J. Clinical experience in the management of pulmonary opportunistic infection and rejection in recipients of heart-lung transplants. Thorax 1988;43:7629.

8 Otulana BA, Higenbottam TW, Scott JP, Clelland C, Hutter J, Wallwork J. Pulmonary function monitoring allows diagnosis of rejection in heart-lung transplant recipients. Transplant Proc 1989;21:2583-4

9 Otulana BA, Higenbottam TW, Scott JP, Clelland C, Igboaka $\mathrm{G}$, Wallwork J. Lung function associated with histologically diagnosed acute lung rejection and pulmonary infection in heart-lung transplant patients. Am Rev Respir Dis 1990;142:329-32.

10 Hoeper MM, Hamm M, Schäfers HJ, Haverich A, Wagner TOF. Evaluation of lung function during pulmonary rejection and infection in heart-lung transplant patients. Chest 1992;102:864-70.

11 Van Muylem A, De Vuyst P, Yernault JC, Paiva M. Inert gas single-breath washout and structural alteration of respiratory bronchioles. Am Rev Respir Dis 1992;146:116772 .

12 Van Muylem A, Antoine M, Yernault JC, Paiva M, Estenne $M$. Inert gas single-breath washout after heart-lung transplantation. Am Ұ Respir Crit Care Med 1995;152:947-52.

13 Yousem SA, Berry GJ, Brunt EM, Chamberlain D, Hruban $\mathrm{RH}$, Sibley RK, et al. The working formulation for the standardization of nomenclature in the diagnosis of hear and lung rejection. F Heart Lung Transplant 1996;15:1-15.

14 Paiva M, Van Muylem A, Ravey P, Yernault JC. Inspired volume dependence of the slope of the alveolar plateau. Respir Physiol 1984;56:309-25.

15 Swets JA, Pickett RM, Witchead SF, Getty DJ, Schnur JA Swets JB, et al. Assessment of diagnostic technologies. Science 1979;205:753-9.

16 Quanjer P. Standardized lung function testing. Bull Eur Physiopathol Respir 1983; 19 (Suppl. 5):7-10. 